\title{
TOPICS
}

Topics in Cognitive Science (2017) 1-18

Copyright (C) 2017 Cognitive Science Society, Inc. All rights reserved.

ISSN:1756-8757 print / 1756-8765 online

DOI: $10.1111 /$ tops.12264

This article is part of the topic "Rumelhart Issue Honoring Dedre Gentner," Jeffrey Loewenstein and Arthur Markman (Topic Editors). For a full listing of topic papers, see http://onlinelibrary.wiley.com/journal/10.1111/(ISSN)1756-8765/earlyview

\section{Structure Mapping for Social Learning}

\author{
Stella Christie \\ Department of Psychology, Swarthmore College
}

Received 24 June 2016; received in revised form 12 December 2016; accepted 11 January 2017

\begin{abstract}
Analogical reasoning is a foundational tool for human learning, allowing learners to recognize relational structures in new events and domains. Here I sketch some grounds for understanding and applying analogical reasoning in social learning. The social world is fundamentally characterized by relations between people, with common relational structures - such as kinships and social hierarchies - forming social units that dictate social behaviors. Just as young learners use analogical reasoning for learning relational structures in other domains-spatial relations, verbs, relational categories - analogical reasoning ought to be a useful cognitive tool for acquiring social relations and structures.
\end{abstract}

Keywords: Analogy; Comparison; Cognitive development; Learning; Relational thinking; Social cognition

\section{Introduction}

Interactions in the social world are shaped by relations between people. On the most basic level, learning about the social world necessitates understanding different kinds of relations because relations dictate social expectations and behaviors. One demands love and care from one's parents, one helps and expects help from friends though not from enemies. On a higher level, many types of relations between people cohere to form complex structures: parents and children comprise a family, whereas friends, classmates, and

Correspondence should be sent to Stella Christie, Department of Psychology, Swarthmore College, 500 College Ave., Swarthmore, PA 19081. E-mail: christie@swarthmore.edu 
teachers form a school hierarchy. Combining the individual relations and relational structures, it is clear that navigating the social world requires exploiting relational commonalities-relations and structures that hold across space and time. Indeed, understanding a singular token of the friend relation does not advance our understanding of the social world; instead, the relation friend becomes useful when we recognize how different people relate to us as friends and observe when other people hold this relation among themselves. Similarly, grasping the school-hierarchy structure, complete with its predictions and consequences, is most useful when we perceive it across different times in our social life.

In sum, the crux of social learning is in understanding relations and abstracting common relational structures. Since relations and relational commonalities are precisely what concerns analogical learning, it is logical to expect that analogical mechanisms play an important role in social learning. In this paper, I outline how the Structure Mapping Theory (SMT; Gentner, 1983) is relevant for social development-how structure mapping can aid the acquisition and perception of social relations and commonalities. Recent work has demonstrated the importance of structural alignment for Theory of Mind (ToM) learning (Hoyos, Horton, \& Gentner, 2015), goal understanding (Gerson, 2014; Gerson \& Woodward, 2012), and selective learning and pedagogy (Christie, Fialkow, \& Lastarria, 2015). By situating these developments within a larger framework of analogical reasoning, I aim to lay a foundation for a new research program, aimed at understanding how the structure mapping process shapes and predicts social learning.

\section{The learning problems of social relations}

\subsection{Knowledge of relations}

The developmental analogy literature documents extensively that learning relations is challenging. For example, children take uncle to initially mean a man who has a moustache and smokes a pipe rather than a sibling of one's parents (Keil, 1989). Preschoolers who were taught a new relational term like passenger took it to mean an individual person rather than a (relationally defined) rider of a conveyance (Waxman \& Hall, 1993). As these examples show, rather than focusing on the relations, children find the apparent perceptual features more salient. The most basic cause of this difficulty is that children may not yet know the relation in question (but are able to perceive the perceptual features). If you do not have the concepts of symmetry and proportion, staring for hours at a Mondrian painting will not help you to see in it anything beyond an arbitrary collection of red, yellow, and blue blocks.

Charting the initial stock of social relational knowledge-which social relations children acquire early on (mother, father, or friend?) and which are learned later-will be useful for understanding the initial drives and developmental directions of socio-cognitive learning. This is because children's initial relational knowledge shapes their perception of and hypotheses for the workings of the social world. For example, a child who has 
acquired the relational concept friend but not foe may categorize people solely based on the friend relation and, as a consequence, be more likely to help others and expect them to share toys and resources with her. In contrast, a child who commands both the relations friend and foe may distribute her attitudes and expectations more prudently.

\subsection{Relational versus object similarity}

But knowledge of relations by itself is often not sufficient for analogical insights. Infants may already know the relation identity, but even 3-year-olds have difficulty in perceiving the relational similarity between two instances of the identity relation (Christie \& Gentner, 2014). A distinct cognitive step separates knowing relations and being able to abstract and generalize based on that knowledge. In later sections I discuss in detail how comparison and language foster this learning. But first, let us understand some implications of the challenging nature of relational similarity.

Pertinent to social learning, we may see a parallel learning gap-where early on infants perceive some basic social relations but learn to abstract commonalities across events and situations only after a delay. The progression between knowing relations and abstraction of relational commonalities probably takes a unique path in social learning because children perceive social relations as relevant and salient from very early on. For example, Johnson, Dweck, and Chen (2007) showed that 12-16-month-old infants have different expectations of behavior depending on the attachment relations between infants and caregivers. Securely attached infants were surprised to see a "mother" showing unresponsive behavior to a "child" (the mother and the child were animated geometric characters), whereas insecurely attached infants did not show surprise at this unresponsive event. This illustrates that the type of attachment (secure or insecure) shapes infants' expectations of the caregiver-child pair. The question is how far this abstraction applies: Will infants perceive a relational commonality across many forms of caregiver-infant pairs? A subsequent development in social relational learning demands that learners not only know the relation they themselves have experienced (e.g., a responsive relation for the securely attached infants), but also identify different types of relations across many social entities.

Perceiving relational commonality can also be difficult because children find object commonality more salient than relational commonality. Four-year-olds confronted with a relational similarity matching task tend to ignore the relational match, favoring the object match instead (given AA, chose AB instead of CC; Christie \& Gentner, 2007). Older children who knew many relations also found object matches more salient than the relational ones. For example, in a scene analogy task, 4-year-olds could easily describe the scene of a cat chasing a mouse, but when asked what the cat goes with in another chasing scene (boy chasing girl), they did not pair cat-the-chaser with boy-the-chaser. Instead, children simply matched the cat in the first scene to another cat in the second scene (Richland, Morrison, \& Holyoak, 2006), favoring an object match over the relational match. 
I suggest that the tension between relational and object similarity is also pertinent to social learning, though in a more nuanced way than in other cognitive domains. In the social world, the "object match"-type similarities may include sex and age, likely favored over the more relational categories such as cooperation. If the object-based grouping is indeed more salient than relationally based grouping, it would have potentially far-reaching consequences for children's social inferences. As a possible effect, children might predict that people who share surface features are more likely to have common goals than people linked by a more abstract relation. On the other hand, children who know the friend relation can surely predict that two friends are more likely to share a common goal than two similar-looking non-friends. Without empirical input, we may only say that social relations, with their manifold manifestations and degrees, may play out against the more superficial social similarities in a number of different ways. Yet up to now, no developmental studies have ever pitted relational matches against object matches in social category learning - despite the prevalence of such studies in non-social domains. Such work would be an essential step toward mapping the social learning patterns and understanding human analogical learning in general.

The analogical development literature also reports that children only initially focus on object matches, with a subsequent shift toward relational matches. This relational shift (Gentner, 1988; Halford, 1987, 1992; Rattermann \& Gentner, 1998) is robust but does not depend on the maturational process. Rather, the shift occurs depending on domain knowledge: a greater relational knowledge makes for a greater likelihood of focusing on relations rather than on objects. Thus far, one study has reported that adults favor relational commonalities over object commonalities when selecting social comparison standards. Mussweiler and Gentner (2007) asked adult participants to choose between a standard who shared many features but did not share structure and a standard who shared connecting structures but differed in all specific features. For example, if Bob (the target) was a sophomore highly dedicated to sport, the feature-match standard Adam shared many surface commonalities (same gender, disinterest in cultural events, athletic build), whereas the relational-match standard Melissa shared only similar structures (a high dedication to some pursuit, say, music) without common surface features (different gender, interests, hobbies). The results showed that participants preferred to choose Melissa-therelational-match standard over Adam-the-feature-only-match as the appropriate social comparison standard for the target. These findings are not likely to extend to young children, who probably start out favoring feature-matching social comparison standards instead. Considering its enormous social consequences, it is imperative to investigate the social relational shift-when and how it occurs, what drives it, what social tasks it affects.

\subsection{The social learning problem-summary of questions}

1. What is the stock of social relational knowledge? Which social relations do children know early on and which ones are acquired later? 
2. How does social relational knowledge affect social behavior and perception of others?

3. How do children generalize social relations?

4. Do learners initially favor object over relational commonality in social category learning?

In the next section I discuss the learning tools that have been useful in promoting analogical insights in other domains and how the same learning tools are relevant for social learning.

\section{Alignment for abstracting social relations}

Structural alignment results in relational abstraction. Learners who align two (or more) exemplars perceive relational commonalities between the two exemplars which are not available or salient prior to comparison. Beyond simply highlighting relational commonalities, the act of aligning can be a springboard for learning entirely new concepts. Indeed, we found that 4-year-olds who compared two exemplars of arbitrary new relations such as "black-on-top-white-at-the-bottom" successfully learned this novel relation. But 4-yearolds who saw only one exemplar, or even two exemplars without aligning them (seeing them sequentially), did not learn the new relational concept (Christie \& Gentner, 2010).

These findings are relevant for social learning where learners must constantly acquire and abstract new social relations. I highlight two areas of social learning, in which alignment was recently shown to play a crucial role for abstracting and generalizing social relations.

\subsection{Goal and intention learning}

As social beings, we perceive the relations between actors and objects (such as a hand extending for a cup) not merely as physical descriptions (hand-cup), but as intentional relations (a hand wanting a cup) (Woodward, 1998). Collapsing the myriad of physical events that happen within a learner's life into a compact set of intentional relations renders the world manageable. A hand almost on the cup handle, a hand grasping the cup's handle, only two fingers grasping the cup handle - all can be simplified into a single intentional relation, rather than encoded as minute details of physical events.

A large body of research has shown that infants are able to abstract physical events into intentional relations (see Woodward, Sommerville, Gerson, Henderson, \& Buresh, 2009, for review) and that this is a learned ability: The process of abstraction develops over time. One example is that by 6 months infants perceive hands reaching an object as an intentional relation - the hand "wants" the cup, as opposed to simply approaching its physical location (Woodward, 1998). But infants under 12 months do not perceive the goal aspect of the same event when it involves tool use (e.g., Cannon \& Woodward, 2012; Sommerville \& Woodward, 2005). Such specialization (hands privileged over tools) 
is likely useful in the early learning process. But eventually one must be able to extend intentional relations even to tools, so as to process the many instances of tools mediating human intentions such as tongs picking up meat off a grill.

Gerson and Woodward (2012) asked if 7-month-old infants could generalize the intentional relation to a claw after aligning their own reach with a tool's reach. In the actionalignment condition children saw a tool reaching one of two objects aligned with their own hands reaching for the same object, whereas the control group saw the same tool reaching for the same object but not aligned with the infant's reaching action. When shown a new event of the tool reaching for another object, infants in the alignment group generalized the intentional relation to tool-reach - several months earlier than the normal learning course - whereas infants in the control group did not. The alignment process is efficient; infants only need to see several (ideal) alignment events to get to a point that otherwise takes several months. The alignment process is also crucial; seeing the same events (tool reach, own hand reach) without alignment did not result in learning. This agrees with prior research showing that children who saw the same number of exemplars without aligning them do not learn the relational commonalities (e.g., Christie \& Gentner, 2010).

\subsection{Alignment for ToM learning}

Several researchers have posited that the process of alignment can foster the development of ToM (Bach, 2014; Baldwin \& Saylor, 2005; Pham, Bonawitz, \& Gopnik, 2012; San Juan \& Astington, 2012). While infants have been shown to have an early understanding of ToM (see Baillargeon, Scott, \& He, 2010 for a review), there is ample evidence that this ability develops between 3 and 5 years of age (Wellman, Cross, \& Watson, 2001). Thus, a full-blown ToM requires learning; the question is what kind of learning aids ToM development. Gopnik and Wellman's (1992) theory-theory approach posits that this learning entails constant revisions of one's theory about other minds; every time the learner is confronted with evidence inconsistent with her current theory, she revises her theory. Here is where the process of alignment can be useful: Good alignment better highlights similarities and differences, allowing learners to identify how their own thoughts differ from others or observe that two people may have different beliefs about the same event. Learners then need to abstract this difference relation across people's thoughts and realities. It is not sufficient to understand a single instance of Jane thinking the cereal box contains cereal while in reality it contains crayons. Rather, having a ToM entails the understanding that Jane's (and everyone else's) thoughts could differ from reality. Since alignment highlights relational commonalities, aligning people's thoughts to one another or to one's own can bring about this abstraction.

Recent evidence supports the hypothesis that structural alignment can bring about ToM learning, in particular the understanding of false belief. Hoyos et al. (2015) presented 4.5to 5-year-olds with comparisons of characters and their thoughts, contrasting true and false beliefs in an unexpected content situation. Children saw two cereal boxes, one containing and one not containing cereal. Both characters thought that the cereal boxes 
contained cereal (their thoughts represented in thought bubbles), and children were asked questions that allowed them to align the characters' thoughts with the actual content of the boxes. After this Compare Thoughts training, children were tested in several falsebelief tasks (posttest) and their performance was compared to the pretest. To test the usefulness of structural alignment in particular, two control groups were included in the study: a Compare Items group, where children compared the two characters and their items but not their thoughts, and a Baseline group, where children went directly from the pretest to the posttest without any training.

The results showed that only children who explicitly compared mental states made a significant gain in their false belief posttest. Those who simply compared characters and items did not; neither did the Baseline group. Comparison by itself is not sufficient; children had to align the relations between characters' thoughts and realities in order to gain relational abstraction. Remarkably, this gain of false belief understanding can be made within the course of a single experiment with simple alignment training.

In the above examples, even a brief invitation to align allowed children to make remarkable gains in learning intentional relations and the ToM. But in everyday learning, such ideal forms of alignment are probably rare. To postulate that the process of alignment is feasible and relevant in everyday learning, we must understand when it happens spontaneously. Analogical comparison research has identified two catalysts that can invite alignment: (a) initial high similarity-similar-looking things or events naturally invite comparison, even when learners cannot initially see the relational similarity; (b) language - a common label invites learners to compare the events to which it refers.

In the following I discuss how each of these factors can play a role in inviting comparison in everyday social learning.

\subsection{Similarity invites initial social alignment}

Young learners find object matches salient (e.g., Rattermann \& Gentner, 1998; Richland et al., 2006; Son, Smith, \& Goldstone, 2011). In analogy tasks where object and relational matches are pitted against each other, for example in the relational match to sample task (sample AA, choices $\mathrm{BB}$, or AC), a strong preference for object matches (choosing AC) can impede perception of relational similarity (choosing BB) (Christie \& Gentner, 2007). However, this spontaneous attention to object similarity also comes with a learning benefit: Even without an explicit comparison prompt, young children who easily notice a cat matching another cat can spontaneously compare them. Since comparison highlights common relational structure (Christie \& Gentner, 2010; Gentner \& Namy, 1999; Kotovsky \& Gentner, 1996), easy initial similarity serves as an invitation to align, which eventually results in relational learning. Importantly, because this invitation to align rests on children's spontaneous ability to notice object matches, it is relevant in everyday settings.

Hoyos et al. (2015) showed that similarity indeed matters for 4.5-year-olds learning about false beliefs. They contrasted two comparison conditions: A High-Similarity condition where children compared similar characters and objects across three scenarios (two 
true-belief scenarios followed by a false belief scenario) and a Low-Similarity condition where children compared across the same three belief scenarios but with different characters and objects. To test the feasibility of spontaneous comparison, children were given very minimal explicit comparison prompts and simply observed the three belief scenarios. The results showed that despite getting the same opportunity to observe true and false belief events, only children in the High-Similarity condition performed well in post (final) false-belief tests. Extrapolating this result to everyday learning, whereas learners are typically confronted with diverse false belief events, when the events contain an object similarity - mom thought it was raining but it was not, and mom thought the shoes were in the closet but they were not-children are spontaneously called to compare the events. As a consequence, they may be able to abstract their relational commonality and arrive at an understanding of false beliefs.

This ease of comparison may also be the reason why young children learn to abstract intentional actions best when they are themselves involved in the alignment process. Gerson and Woodward (2012) showed that 7-month-old infants extended intentional relations to non-agents like metal claws only after seeing an alignment between self reaching for a toy and a metal claw reaching for the same toy. But when infants observed the same alignable events of another person and a tool, they did not generalize the intentional relation. One possible explanation is that although these events were aligned, the infants did not perceive the alignment. With self-reaching, however, the alignment was manifest.

Self is often involved in the relations defining the social world. The child discovers that many of her beliefs are false, experiences the friend relation in her interactions with peers, and calls someone my grandmother. The frequent self-involvement can act as object similarity - the self is the same across different events. The re-occurrence of self invites alignment, resulting in relational abstraction. Connectedly, Meltzoff's Like Me hypothesis (Meltzoff, 2005) postulates that learners need to align themselves to others before they are able to imitate others' actions. We see this self-other alignment even in newborns' imitation (Meltzoff \& Moore, 1983). Thus, easy similarity involving self invites the child to learn by comparison throughout development.

Similarly, extensive works by Tomasello and colleagues show that young children readily take on social roles. They spontaneously help others (Warneken \& Tomasello, 2006), cooperate (Tomasello \& Vaish, 2013; Warneken, Chen, \& Tomasello, 2006), even arbitrate others who do not follow social norms (Rakoczy, Warneken, \& Tomasello, 2008). By frequently playing out these roles across many different contexts, young children have ample opportunities to do alignment, resulting in abstraction of common relational structure. For example, little Joe who has helped Mom to put away toys and open the door can align these events and learn the common "helper-helpee" relation. Such learned abstraction begets further learning about relations, for example, knowing the relation "helper" or "cooperator" makes it easier to understand the absence of these relations — "non-helpers" and "non-cooperators." Learners can then distribute actions appropriately based on this relational understanding - such as punishing the non-cooperators (Vaish, Hermann, Markmann, Tomasello, 2016). Indeed, in recent years we have seen an explosion of works showing children's precocious social ability and behavior (for a review see 
Tomasello, 2014). A logical and interesting next step is to investigate the learning mechanisms that bring about this precocious social development. Self-initiated alignment could be a relevant mechanism.

Besides self, the social entities that populate a child's life are initially limited to a few characters, so the same people are likely to be involved in multiple social relations. Thus, the social world is rife with object similarities that invite alignment and subsequent comparison, perhaps more so than other domains like numerical or spatial relations. If so, one intriguing prediction is that children's relational thinking may be particularly advanced in the social domain. This prediction is currently untested. If true, it could open an interesting direction in analogical research. For example, we may postulate that children first learn about many relations when they are substantiated in the social domain (such as biglittle between people) and only later extend the said relation to other domains.

\subsection{Language invites social comparison}

Learners who hear two or more events called by the same label are naturally prompted to compare them, which highlights their relational commonalities. For example, children hearing two action events labeled by the same verb may learn the relational commonality (the verb meaning) despite seeing different agents and recipients across the events (Childers \& Paik, 2009; Childers et al., 2016; Pruden, Hirsh-Pasek, Shallcross, \& Golinkoff, 2008). This mechanism - that a common label invites comparison-is distinct from another role of language in relational learning, whereby known relational labels make specific relational concepts more salient (see Gentner \& Christie, 2010; Gentner \& Namy, 2006, for a full explication of this relational label mechanism). Instead of relational labels, in this mechanism any label can invite comparison, regardless of whether or not young children know the label's meaning, as long as they have the assumption that like names refer to like things and relations. In Christie and Gentner (2014), 3-year-olds who were reminded about the word same prior to being tested in a Relational-Match-to-Sample task (sample AA, match to $\mathrm{BB}$ not $\mathrm{CD}$ ) perceived the relational matches of identity relation, but 2-year-olds did not. Further tests showed that 2-year-olds did not have a good understanding of the label same, which was why they did not reap the relational language benefit. But when 2-year-olds heard common novel labels, they succeeded in perceiving the relational matches. The novel label did not make the relational concept salient (they had no a priori meaning), but they invited comparison that highlighted relational commonalities.

The process whereby unfamiliar common labels invite comparison probably happens extensively in social learning. Social relational labels are complex and many. Take kinship terms; it will take some time for young children to understand their relational content. Yet children do use and hear these terms regularly and may be invited to compare when they hear "my aunt" versus "Sophie's aunt" or "my aunt Amy" versus "my aunt Anne." Such comparison eventually makes them realize that "aunt" does not refer to a specific person and brings to focus the relational meaning of "aunt." 


\subsection{Cultural linguistics: Specific versus general terms}

Several interesting learning challenges arise from this hypothesis. First, in many cultures certain kinship terms are used rather generally; for example, "aunt" and "uncle" function as salutation forms for any males and females of one's parents' age, including strangers. As another example, in China, partly due to the one-child policy, young children often call their cousins or children of close family friends sister and brother. Such proliferation of usages yields plenty of opportunity for comparison. Yet the results of the learning may differ relative to a culture where the kinship terms are used exclusively for actual family relations. Children from a culture with the more relaxed usage may learn the terms earlier but have a less precise understanding of their relational content. In contrast, children from cultures with the more restricted usage may learn the terms later but more precisely. There is room here for specific, cultural-linguistic predictions based on the magnitude of the comparison (learning) pool and the coherence of comparison results.

There are also opposite cultural-linguistic environments, where kinship terms are very specific. For example, some languages have different terms for maternal and paternal uncles, grandfathers, grandmothers, etc. Such specificity derives opposite predictions from the two language-relational learning mechanisms discussed above (familiar relational labels vs. any common labels). Focusing on the effect that relational labels reify relational concepts, children growing up in ultra-specific kinship environments have access to many more labels that highlight more specific relations. One may therefore predict that Polishspeaking children (who use wujek and stryjek for maternal and paternal uncle, respectively) will learn these relations better than children learning English (who learn only one term uncle for both categories). On the other hand, the more restrictive usage means that the Polish children encounter a smaller incidence of invitations to compare. Indeed, considering the second language pathway-unknown common labels invite comparisonmakes manifest that the Polish children miss out on comparing maternal and paternal uncles as their English-speaking peers are invited to do.

An empirical account of relational learning pathways (naming relations vs. inviting comparison) across different kinship environments would be fascinating. Similar investigations have been carried out in other domains; see, for example, Sarnecka, Kamenskaya, Yamana, Ogura, and Yudovina (2007) for a comparative study of the acquisition of numbers depending on the specificity of the numeral system of one's language. But regarding kinship terms and social relational concepts, no such comparative data are currently available.

\section{Alignable differences}

Structure Mapping Theory posits that comparison involves a process of structural alignment. The alignment process goes beyond noticing common features; it also identifies common relations that connect the features. Here a natural corollary arises: By noticing a similarity of structures, learners should also notice Alignable Differencesdifferences between corresponding positions in the respective structures. As an example, 
take the following two scenes: a cat chasing a mouse and a boy chasing a dog, watched by mom. Cat-the-chaser and Boy-the-chaser play analogous roles, so the difference between them is alignable. The difference between cat and mom, however, is a Nonalignable Difference - because they do not occupy corresponding roles. SMT predicts that learners favor Alignable Differences over Nonalignable Differences; they find the former more salient than the latter. Because high-similarity pairs allow for easier alignment, SMT also predicts that, paradoxically, identifying differences should be easier in highsimilarity comparisons.

These predictions have been confirmed in non-social domains. Gentner and Markman (1994) gave adult participants a page full of word pairs and asked them to find as many differences as possible within limited time. They found that participants listed a greater number of alignable differences among high-similarity word pairs than among low-similarity word pairs. Similar results were obtained when adults inspected pairs of images rather than words (Markman \& Gentner, 1996), a pattern consistent with another study by Sagi, Gentner, and Lovett (2012). Given Fig. 1, adults who were asked to compare A and $\mathrm{B}$ took less time to identify the differences than those who compared $\mathrm{A}$ and $\mathrm{C}$. The A-B pair shares more common features and structures, so their difference is more alignable and therefore more noticeable than the differences separating A from C.

It is worth stressing that we are focusing here not on any kind of differences, but on alignable differences. Of course, it is faster to notice that $\mathrm{A}$ and $\mathrm{C}$ are simply different from each other than to do so with A and B (confirmed in the Sagi et al., 2012, results). However, the difference(s) that separates A from B is arguably more meaningful and portable. Supporting this, Gelman, Raman, and Gentner (2009) asked 4-year-olds and adults to list as many differences as they could, now without time limit. Both children and adults listed more "deep differences" (actions, functions, traits, mental states) for highsimilarity pairs (such as spoon/fork) than for low-similarity pairs (spoon/dog).

In the social domain, noticing alignable differences in high-similarity comparison may potentially play a large role in explaining diverse social learning phenomena. For example, the similarity-difference paradox may explain why we feel most competitive when we compare ourselves to similar others than to dissimilar others. On a larger scale, deep social and political enmities between cultures or nations often afflict pairs of close and similar rather than distant entities. It is possible that the underlying mechanism is precisely the highlighting of meaningful alignable differences, ushered in by similarityinduced comparison.

Below I lay out a number of emerging areas in social learning where alignable differences implicitly play a role. I also chart several suggestions for how to explicitly test the alignable difference hypothesis.

\subsection{Alignable difference in social comparison}

Festinger's social comparison theory (Festinger, 1954) captures the notion that "high similarity begets alignable differences." One of Festinger's most important hypotheses is that we tend to compare ourselves to similar others rather than to dissimilar others. 
(A)

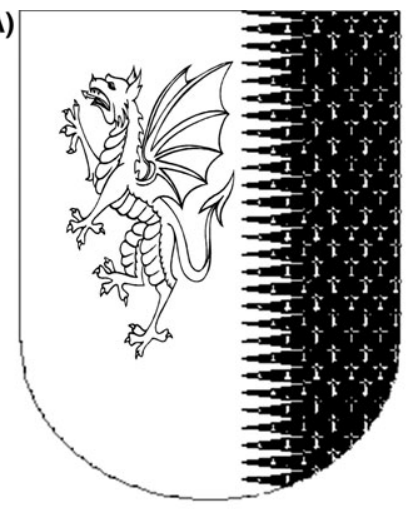

(C)

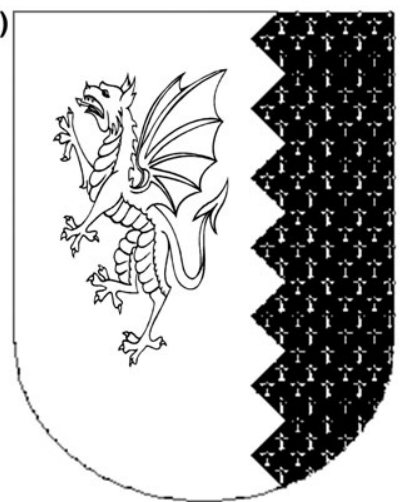

(B)

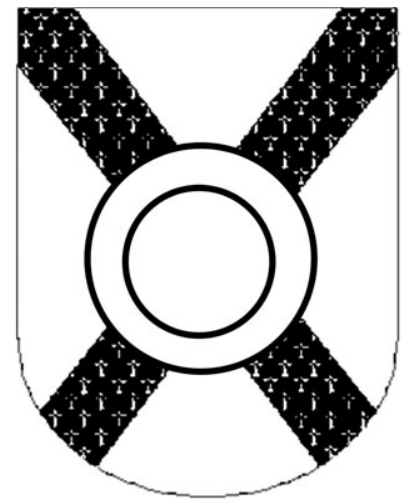

(D)

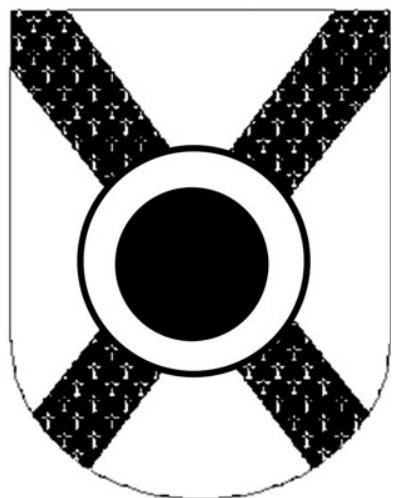

Fig. 1. Similarity comparison of pairs A and B results in an Alignable Difference, whereas comparison of non-similar pairs A and C (or B and D) results in Nonalignable Differences. From Sagi et al. (2012).

Comparing to similar others is favored because it gives a more useful metric to evaluate one's ability. If Joe wants to know whether he is a good tennis player, it is sensible to compare himself to Matt, who is of the same age, holds a similar job (and therefore has a similar work-play time division), and has been playing tennis for a similar amount of time; comparison to Serena Williams is probably less telling. The differential utility of the two comparisons reflects the greater alignability of Joe and Matt's circumstances, which in turn originates from the fact that they are more similar than Joe and Serena are. A robust body of evidence supports the similarity-comparison hypothesis in adults (Corcoran, Crusius, \& Mussweiler, 2011; Goethals \& Darley, 1977; Wood, 1989). Moreover, Muesweiller and Gentner (2007) showed that, consistent with the SMT, adults prefer to compare themselves to others who are structurally similar rather than to those who merely share feature commonalities.

Self-to-others comparison is one of the most salient socio-cognitive hallmarks in development. It is generally noted that children progress from non-evaluative self-comparison 
("I'm the best runner") to judgmental self-other comparison ("I'm a better runner than my friend" between age 4 and 8 years. But up to now, little investigation has been aimed at charting the cognitive mechanisms which bring about the onset of comparison. This is despite the fact that self-to-others comparative evaluation has deeply important social and cognitive consequences, such as the formation of identity and self-confidence. Understanding the relevant developmental mechanisms would be very beneficial.

Structural alignment may be a useful tool in this regard. First, the onset of comparison can be initiated by (a) high-similarity and (b) common labels. Children surrounded by similar others - peers in preschools, a twin sibling, growing up in a large family household with same-age cousins - have more initial invitations to compare than children with scarcer access to similar others. Common labels applied to activities- "Emma just finished eating her snack, and you should finish yours too"-may also prompt children to align. Second, in the development of self-to-others comparison, children should (a) prefer comparing themselves to highly similar than to less similar others, and (b) initially find mere feature similarity more salient, with an eventual shift to structural similarity. That is, little Joe should prefer comparing himself to the highly similar Matt from the same class rather than to Jane from another class. But as Joe takes up violin and Jane spends substantial time in soccer practice, the closer make-up of interests and time allocation may render Jane the more salient comparison target than the superficially similar Matt.

\subsection{Alignable difference in selective social learning}

While Festinger's social comparison focuses on how we compare ourselves to others to learn about ourselves, a large portion of social comparison includes comparison between others. We often evaluate who among our friends is the most trustworthy, who among our mentors gives the best career advice, or which among our foes is most likely to give us trouble. Such judgments are immensely useful in making informative decisions about whom to trust or whom to avoid - allowing us to selectively learn from others.

The act of comparing and selecting others naturally adds salience to alignable differences between people. Intuitively, we find differences most meaningful when they pertain to alignable dimensions. We notice that Jane has healthy eating habits, whereas Joe indulges his sweet tooth, but do not remark that Jane eats healthily while Joe likes modern art. Structural alignment theory captures this intuition: Comparing highly similar and alignable pairs highlights alignable differences. This principle is implicitly used in many developmental studies showing that children evaluate others and learn from them selectively. For example, children who were shown two teachers - one who named objects correctly versus one who named objects incorrectly - preferred to learn new labels from the historically correct than the incorrect teachers (Harris \& Corriveau, 2011). Children also preferred to learn from a more confident than a less confident teacher (BrosseauLiard, Cassels, \& Birch, 2014). In evaluating which teacher was better, 4-year-olds were affected by whether the teacher had given full or only partial information on how to play with a toy (Gweon, Pelton, Konopka, \& Schulz, 2014). In all these studies, the 
dimensions in which children evaluated others were well aligned: correct versus incorrect naming, confidence, completeness of information.

It is impressive that children as young as 3 years of age can evaluate and selectively learn. But it is also important to ask whether children employ such selectivity when the informants/teachers are not a priori well aligned. For example, if instead of correct versus incorrect naming, children see a person who accurately names objects versus one who blunders in counting (a less alignable comparison), will the preference for the correct teacher persist? Or will they want to find out about the relevant dimension of comparison and, given an opportunity, test the wrong counter on a naming task? The underlying theme here is that the social world does not present itself as a ready array of aligned comparisons; instead, learners must actively conduct comparisons and project their results onto a select set of axes. It would be revealing to know whether learners spontaneously generate the most alignable comparisons in evaluating others.

Structural Alignment also gives rise to a paradoxical hypothesis: Comparisons of similar pairs reveal more differences than do comparisons of dissimilar pairs. This gives interesting predictions - currently untested-for social interactions. First, I predict that we are more likely to compare people within one group (especially our own group, which we know best) than to compare people across groups or others from out-groups. As a result, we simultaneously see members of our own group as more similar and more differentbecause we are able to spot a greater number of meaningful differences. This reasoning predicts that children view in-group members to have more diverse personalities than members of out-groups. On a higher level of social interaction, lack of comparison and alignable differences could contribute to group prejudice and stereotyping. By not comparing individuals from another group, one fails to discern that its members can and do differ from one another. The similarity-begets-differences paradox has a potentially strong clarifying power for understanding social dynamics and deserves a more systematic study.

\subsection{Alignable differences for teaching others}

Humans give information to others, sometimes unsolicited. Some theorists have argued that this pedagogical tendency is uniquely human (Csibra \& Gergely, 2009) and underlies our social cognitive learning (Tomasello, Kruger, \& Ratner, 1993). My hypothesis is that comparison plays an important role in pedagogy: We select from our wealth of knowledge those data which are relevant for sharing. The comparison can be explicit (this taquería has better salsas than that one), but it is more often implicit (without mentioning the baseline of less remarkable restaurants).

The principles of structural alignment apply in pedagogical comparison, both in classrooms and in unsolicited information transfer. Classroom research advises mathematics teachers to use similar and alignable solutions in teaching a novel technique (Rittle-Johnson \& Star, 2009). Parents asked how to best teach their children novel adjectives ("blickish") spontaneously use contrasting alignable exemplars (a striped cup vs. a dotted cup, where "blickish" refers to stripes) rather than less alignable contrasts (a stripy cup 
vs. a dotted pencil) (Manders \& Hall, 2002). These findings accord with the well-tested usefulness of similarity and comparison in children's learning of verbs (Childers et al., 2016; Maguire, Hirsh-Pasek, Golinkoff, \& Brandone, 2008) and spatial concepts (Casasola, 2005; Gattis, 2004; Loewenstein \& Gentner, 2001).

Interestingly, when 3-year-old children assume the roles of teachers and instruct naive others, they also spontaneously reach for alignable contrasts. This is observed in a preliminary study in my lab (Christie et al., 2015).). But this only scratches the surface of the larger question - whether and when young children employ structural alignment in conveying information. For example, does children's propensity to use alignable contrasts differ between goal-oriented teaching set-ups and unsolicited sharing of information?

\section{Summary}

Why Structure Mapping for social learning? First, because we see social beings in terms of their relations to others, social learning problems are relational. Using the Structure Mapping approach allows us to parameterize the scope of the social learning problem: what social relations are inherent (if any) and why it is challenging to abstract social relational similarities. Second, Structure Mapping gives us precise tools and hypotheses by which these problems can be solved. Here I charted how (a) comparison, (b) similarity, (c) mutual bootstrapping between language and comparison, and (d) alignable differences are relevant in social cognitive learning. This paper is only partly a review of previous studies done on the subject; in a larger part, it charts studies that ought to be done in the future.

\section{Acknowledgments}

I thank Bartłomiej Czech, Elizabeth Spelke, Hyoweon Gweon, Dedre Gentner, and members of the Swarthmore Kid Lab for illuminating discussions on the ideas compiled in this paper. I also thank Jeff Loewenstein, Micah Goldwater, and the anonymous reviewer for useful suggestions for the paper. This work is supported by the Swarthmore College Faculty Research grant and the Lang Sabbatical Fellowship.

\section{References}

Bach, T. (2014). A unified account of general learning mechanisms and theory-of-mind development. Mind \& Language, 29(3), 351-381. doi:10.1111/mila.12055.

Baillargeon, R., Scott, R. M., \& He, Z. (2010). False-belief understanding in infants. Trends in Cognitive Sciences, 14(3), 110-118. doi:10.1016/j.tics.2009.12.006.

Baldwin, D. A., \& Saylor, M. (2005). Language promotes structural alignment in the acquisition of a theory of mind. In J. Baird, \& J. Astington (Eds.), Why language matters for theory of mind (pp. 123-143). Cambridge, MA: MIT Press. 
Brosseau-Liard, P., Cassels, T., \& Birch, S. (2014). You seem certain but you were wrong before: Developmental change in preschoolers' relative trust in accurate versus confident speakers. PLoS ONE, 9 (9), e108308. doi:10.1371/journal.pone.0108308.

Cannon, E. N., \& Woodward, A. L. (2012). Infants generate goal-based action predictions. Developmental Science, 15(2), 292-298. doi:10.1111/j.1467-7687.2011.01127.x.

Casasola, M. (2005). When less is more: How infants learn to form an abstract categorical representation of support. Child Development, 76(1), 279-290. doi:10.1111/j.1467-8624.2005.00844.x.

Childers, J. B., \& Paik, J. H. (2009). Korean-and English-speaking children use cross-situational information to learn novel predicate terms. Journal of Child Language, 36(1), 201-224. doi:10.1017/ s0305000908008891.

Childers, J. B., Parrish, R., Olson, C. V., Burch, C., Fung, G., \& McIntyre, K. P. (2016). Early verb learning: How do children learn how to compare events? Journal of Cognition and Development, 17(1), 41-66. doi:10.1080/15248372.2015.1042580.

Christie, S., Fialkow, D., \& Lastarria, W. (2015). Selective pedagogy: children choose the best comparison to teach others. Paper presented at the Biennial Meeting of Society for Research in Child Development, Philadelphia, PA.

Christie, S., \& Gentner, D. (2007). Relational similarity in identity relation: The role of language. In S. Vosniadou, D. Kayser, \& A. Protopapas (Eds.), Proceedings of the Second European Cognitive Science Conference. Taylor and Francis.

Christie, S., \& Gentner, D. (2010). Where hypotheses come from: Learning new relations by structural alignment. Journal of Cognition and Development, 11(3), 356-373. doi:10.1080/15248371003700015.

Christie, S., \& Gentner, D. (2014). Language helps children succeed on a classic analogy task. Cognitive Science, 38(2), 383-397. doi:10.1111/cogs.12099.

Corcoran, K., Crusius, J., \& Mussweiler, T. (2011). Social comparison: Motives, standards, and mechanisms. In D. Chadee (Ed.), Theories in social psychology (pp. 119-139). Oxford, UK: Wiley-Blackwell.

Csibra, G., \& Gergely, G. (2009). Natural pedagogy. Trends in Cognitive Sciences, 13(4), 148-153. doi:10. 1016/j.tics.2009.01.005.

Festinger, L. (1954). A theory of social comparison processes. Human Relations, 7(2), 117-140. doi:10.1177/ 001872675400700202 .

Gattis, M. (2004). Mapping relational structure in spatial reasoning. Cognitive Science, 28(4), 589-610. doi:10.1207/s15516709cog2804_4.

Gelman, S. A., Raman, L., \& Gentner, D. (2009). Effects of language and similarity on comparison processing. Language Learning and Development, 5(3), 147-171. doi:10.1080/15475440902824079.

Gentner, D. (1983). Structure-mapping: A theoretical framework for analogy. Cognitive Science, 7(2), 155170. doi:10.1016/s0364-0213(83)80009-3.

Gentner, D. (1988). Metaphor as structure mapping: The relational shift. Child Development, 59, 47-59, doi: $10.2307 / 1130388$.

Gentner, D., \& Christie, S. (2010). Mutual bootstrapping between language and analogical processing. Language and Cognition, 2(2), 261-283. doi:10.1515/langcog.2010.011.

Gentner, D., \& Markman, A. B. (1994). Structural alignment in comparison: No difference without similarity. Psychological Science, 5(3), 152-158. doi:10.1111/j.1467-9280.1994.tb00652.x.

Gentner, D., \& Namy, L. L. (1999). Comparison in the development of categories. Cognitive Development, 14(4), 487-513. doi:10.1016/S0885-2014(99).00016-7.

Gentner, D., \& Namy, L. L. (2006). Analogical processes in language learning. Current Directions in Psychological Science, 15(6), 297-301. doi:10.1111/j.1467-8721.2006.00456.x.

Gerson, S. A. (2014). Sharing and comparing: How comparing shared goals broadens goal understanding in development. Child Development Perspectives, 8(1), 24-29. doi:10.1111/cdep.12056.

Gerson, S. A., \& Woodward, A. L. (2012). A claw is like my hand: Comparison supports goal analysis in infants. Cognition, 122(2), 181-192. doi:10.1016/j.cognition.2011.10.014. 
Goethals, G. R., \& Darley, J. (1977). Social comparison theory: An attributional approach. In J. M. Suls \& R. L. Miller (Eds.), Social comparison processes: Theoretical and empirical perspectives (pp. 259-278). Washington, DC: Hemispheres.

Gopnik, A., \& Wellman, H. M. (1992). Why the child's theory of mind really is a theory. Mind \& Language, 7(1-2), 145-171. doi:10.1111/j.1468-0017.1992.tb00202.x.

Gweon, H., Pelton, H., Konopka, J. A., \& Schulz, L. E. (2014). Sins of omission: Children selectively explore when teachers are under-informative. Cognition, 132(3), 335-341. doi:10.1016/j.cognition.2014.04. 013.

Halford, G. S. (1987). A structure-mapping approach to cognitive development. International Journal of Psychology, 22(5-6), 609-642. doi:10.1080/00207598708246797.

Halford, G. S. (1992). Analogical reasoning and conceptual complexity in cognitive development. Human Development, 35(4), 193-217. doi:10.1159/000277167.

Harris, P. L., \& Corriveau, K. H. (2011). Young children's selective trust in informants. Philosophical Transactions of the Royal Society of London B: Biological Sciences, 366(1567), 1179-1187. doi:10.1098/ rstb.2010.0321.

Hoyos, C., Horton, W. S., \& Gentner, D. (2015). Analogical comparison aids false belief understanding in preschoolers. In D. C. Noelle, R. Dale, A. S. Warlaumont, J. Yoshimi, T. Matlock, C. D. Jennings, \& P. P. Maglio (Eds.), Proceedings of the 37th annual conference of the Cognitive Science Society (pp. 1-6). Austin, TX: Cognitive Science Society.

Johnson, S. C., Dweck, C. S., \& Chen, F. S. (2007). Evidence for infants' internal working models of attachment. Psychological Science, 18(6), 501-502. doi:10.1111/j.1467-9280.2007.01929.x.

Keil, F. C. (1989). Concepts, kinds, and conceptual development. Cambridge, MA: MIT Press.

Kotovsky, L., \& Gentner, D. (1996). Comparison and categorization in the development of relational similarity. Child Development, 67(6), 2797-2822. doi:10.2307/1131753.

Loewenstein, J., \& Gentner, D. (2001). Spatial mapping in preschoolers: Close comparisons facilitate far mappings. Journal of Cognition and Development, 2(2), 189-219. doi:10.1207/s15327647jcd0202_4.

Maguire, M. J., Hirsh-Pasek, K., Golinkoff, R. M., \& Brandone, A. C. (2008). Focusing on the relation: Fewer exemplars facilitate children's initial verb learning and extension. Developmental Science, 11(4), 628-634. doi:10.1111/j.1467-7687.2008.00707.x.

Manders, K., \& Hall, D. G. (2002). Comparison, basic-level categories, and the teaching of adjectives. Journal of Child Language, 29(04), 923-937.

Markman, A. B., \& Gentner, D. (1996). Commonalities and differences in similarity comparisons. Memory \& Cognition, 24(2), 235-249. doi:10.3758/bf03200884.

Meltzoff, A. N. (2005). Imitation and other minds: The "like me" hypothesis. Perspectives on Imitation: From Neuroscience to Social Science, 2, 55-77.

Meltzoff, A. N., \& Moore, M. K. (1983). Newborn infants imitate adult facial gestures. Child Development, 54, 702-709, doi:10.1111/j.1467-8624.1983.tb00496.x.

Mussweiler, T., \& Gentner, D. (2007). On apples and oranges: Structural alignment in the selection of social comparison standards. Journal of Cognitive Science, 8(1), 1-38.

Pham, K., Bonawitz, E., \& Gopnik, A. (2012). Seeing who sees: Contrastive access helps children reason about other minds. In N. Miyake, D. Peebles, \& R. P. Cooper (Eds.), Proceedings of the 34th Annual Conference of the Cognitive Science Society (pp. 2180-2185). Austin, TX: Cognitive Science Society.

Pruden, S. M., Hirsh-Pasek, K., Shallcross, W. L., \& Golinkoff, R. M. (2008). Foundations of verb learning: Comparison helps infants abstract event components. In H. Chan, H. Jacob \& E. Kapia (Eds.), Proceedings of the 32st Annual Boston University conference on language development (Vol. 2, pp. 402414). Somerville, MA: Cascadilla.

Rakoczy, H., Warneken, F., \& Tomasello, M. (2008). The sources of normativity: Young children's awareness of the normative structure of games. Developmental Psychology, 44, 875-881. doi:10.1037/ 0012-1649.44.3.875. 
Rattermann, M. J., \& Gentner, D. (1998). More evidence for a relational shift in the development of analogy: Children's performance on a causal-mapping task. Cognitive Development, 13(4), 453-478. doi:10.1016/ s0885-2014(98)90003-x.

Richland, L. E., Morrison, R. G., \& Holyoak, K. J. (2006). Children's development of analogical reasoning: Insights from scene analogy problems. Journal of Experimental Child Psychology, 94(3), 249-273. doi:10. 1016/j.jecp.2006.02.002.

Rittle-Johnson, B., \& Star, J. R. (2009). Compared with what? The effects of different comparisons on conceptual knowledge and procedural flexibility for equation solving. Journal of Educational Psychology, 101(3), 529. doi:10.1037/a0014224.

Sagi, E., Gentner, D., \& Lovett, A. (2012). What difference reveals about similarity. Cognitive Science, 36 (6), 1019-1050. doi:10.1111/j.1551-6709.2012.01250.x.

San Juan, V., \& Astington, J. W. (2012). Bridging the gap between implicit and explicit understanding: How language development promotes the processing and representation of false belief. British Journal of Developmental Psychology, 30(1), 105-122. doi:10.1111/j.2044-835x.2011.02051.x.

Sarnecka, B. W., Kamenskaya, V. G., Yamana, Y., Ogura, T., \& Yudovina, Y. B. (2007). From grammatical number to exact numbers: Early meanings of "one," "two," and "three" in English, Russian, and Japanese. Cognitive Psychology, 55(2), 136-168. doi:10.1016/j.cogpsych.2006.09.001.

Sommerville, J. A., \& Woodward, A. L. (2005). Pulling out the intentional structure of action: The relation between action processing and action production in infancy. Cognition, 95(1), 1-30. doi:10.1016/j. cognition.2003.12.004.

Son, J. Y., Smith, L. B., \& Goldstone, R. L. (2011). Connecting instances to promote children's relational reasoning. Journal of Experimental Child Psychology, 108(2), 260-277. doi:10.1016/j.jecp.2010.08.011.

Tomasello, M. (2014). A natural history of human thinking. Cambridge, MA: Harvard University Press.

Tomasello, M., Kruger, A. C., \& Ratner, H. H. (1993). Cultural learning. Behavioral and Brain Sciences, 16 (3), 495-511.

Tomasello, M., \& Vaish, A. (2013). Origins of human cooperation and morality. Annual Review of Psychology, 64, 231-255.

Vaish, A., Herrmann, E., Markmann, C., \& Tomasello, M. (2016). Preschoolers value those who sanction non-cooperators. Cognition, 153, 43-51.

Warneken, F., Chen, F., \& Tomasello, M. (2006). Cooperative activities in young children and chimpanzees. Child Development, 3, 640-663. doi:10.1111/j.1467-8624.2006.00895.x.

Warneken, F., \& Tomasello, M. (2006). Altruistic helping in human infants and young chimpanzees. Science, 31, 1301-1303. doi:10.1146/annurev-psych-113011-143812.

Waxman, S. R., \& Hall, D. G. (1993). The development of a linkage between count nouns and object categories: Evidence from fifteen-to twenty-one-month-old infants. Child Development, 64(4), 1224-1241. doi: $10.2307 / 1131336$.

Wellman, H. M., Cross, D., \& Watson, J. (2001). Meta-analysis of theory-of-mind development: The truth about false belief. Child Development, 72(3), 655-684. doi:10.1111/1467-8624.00304.

Wood, J. V. (1989). Theory and research concerning social comparisons of personal attributes. Psychological Bulletin, 106(2), 231. doi:10.1037//0033-2909.106.2.231.

Woodward, A. L. (1998). Infants selectively encode the goal object of an actor's reach. Cognition, 69(1), 1-34. doi:10.1016/s0010-0277(98).00058-4.

Woodward, A. L., Sommerville, J. A., Gerson, S., Henderson, A. M., \& Buresh, J. (2009). The emergence of intention attribution in infancy. Psychology of Learning and Motivation, 51, 187-222. doi:10.1016/s00797421(09).51006-7. 